Apidologie, 1984, 15 (4), 379-388

\title{
BEWERTUNG DER METHODE ZUR RADIKALEN EINDÄMMUNG DER VARROATOSE
}

\author{
Vladimír VESELÝ und Miloslav PEROUTKA \\ Forschungsinstitut f. Bienenkunde in Dol, 25266 P. Libčice, C.S.S.R.
}

\section{ZUSAMMENFASSUNG}

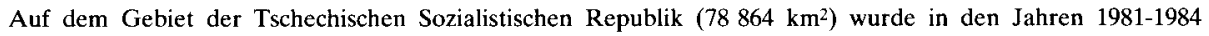
eine radikale Methode zur Eindämmung der Varroatose eingesetzt, die auf einer Abtötung der befallenen sowie der des Befalls verdächtigen Bienenvölker beruht. Ein integrierender Bestandteil der Methodé ist eine beständige gesamtflächige Diagnostik, mit einem möglichst hohen Wirkungsgrad, eine perfekte Erfassung der Bienenvölker und die Liquidierung wildlebender Bienenvölker. Von 1982 an wurden alljährlich alle Bienenvölker auf das Vorhandensein von Weibchen der Varroa jacobsoni $O$. im Wintergemüll untersucht, von 1983 an wurden Bienenvölker im Umkreis von $12 \mathrm{~km}$ von den Herden nach einer chemischen Behandlung mittels $0,01 \%$ iger Wasseremulsion des Akarizids Taktic (Hersteller FBC, England, Gehalt 12,5\% Amitraz) wiederholt untersucht. Die Milbe Varroa jacobsoni O. wurde im Jahre 1978 eingeschleppt, 1981 erstmals diagnostiziert. Bis 1984 (d.h. 6 Jahre nach dem Einschleppen) wurde die Infektion insoweit eingeschränkt, daB sie auf dem gesamten Gebiet bei 1140 Bienenvölkern $(0,17 \%$ des Bienengesamtbestandes) diagnostiziert wurde, wobei die Intensität des Befalls bei der Mehrzahl der befallenen Bienenvölker 10 Varroa-Milben nicht überstieg und keines der befallenen Bienenvölker klinische Symptome der Erkrankung aufwies. In 156 Gemeinden mit Varroatoseherden aus den Jahren 1981-1983 wurde 1984 keine Varroatose mehr verzeichnet. Die Methode ermöglicht eine Minimierung des Einsatzes von Chemotherapeutika und schiebt auch die Entstehung einer Resistenz der Varroa-Populationen hinaus. Es werden weitere Möglichkeiten zur Steigerung der Effektivität des Methode unterbreitet.
\end{abstract}

\section{EINLEITUNG}

Unter dem Begriff der « radikalen Methode » zur Eindämmung der Varroatose verstehen wir die Vernichtung der Milbe Varroa jacobsoni O. sowie ihrer Entwicklungsstadien, zusammen mit dem Wirt, d.h. mit den Imagines und der Brut der Honigbiene. In Europa gibt es den Vorteil, daß die Honigbiene den einzigen Wirt der Varroa $j$. darstellt. Voraussetzung für den Erfolg dieser radikalen Methode ist jedoch eine rechtzeitige, dauerhafte und großflächige Diagnostik von hohem Wirkungsgrad, die objektiv durch Untersuchungsinstitutionen vorgenommen wird, sowie eine einwandfreie Erfassung der Bienenvölker und ein durchgehendes Aufsuchen und Vernichten wildlebender Bienenvölker. 
Die radikale Methode wurde auf dem gesamten Gebiet der Tschechischen Sozialistischen Republik (Č.S.R.) appliziert, d.h. auf einer Fläche von $78864 \mathrm{~km}^{2}$, in mitteleuropäischen Bedingungen (49-51 ${ }^{\circ}$ nördlicher Breite, 150 Tage mit max. Tagestemperaturen unter $+10^{\circ} \mathrm{C}$ ), mit einer hohen und gleichmäßigen Bienenvölkerdichte (erfaßter Evidenzstand zum 1.1.1984 = 686755 Bienenvölker, durchschnittlich 8,7 Völker $/ \mathrm{km}^{2}$ ), mit angeordneter Erfassung der Bienenvölker, mit einer hohen Zahl ( $94 \%$ ) von in Imkersparten organisierten Imkern und mit nur vereinzeltem Vorkommen überlebender wildlebender Bienenvölker.

Die Milbe Varroa jacobsoni O. wurde in die Č.S.R. im Frühjahr 1978 eingeschleppt, u.zw. durch Überführung befallener Bienenvölker in ein etwa in der Mitte der Č.S.R. gelegenes Gebiet. Eine vorherige Invasionsquelle konnte nicht nachgewiesen werden; in keinem der weiteren Herde wurde eine höhere Extensität und Intensität festgestellt. Erstmals wurde die Varroatose im Februar 1981 diagnostiziert, eine gesamtflächige Untersuchung des Wintergemülls aller Bienenvölker wurde im Frühjahr 1982 durchgeführt. Während der drei Saisonen der unkontrollierten Ausbreitung verseuchte die Erkrankung eine Fläche von $1500 \mathrm{~km}^{2}$ rund um das Einschleppungszentrum.

Durch Bienentransporte wurden weitere 11 voneinander getrennte Gebiete mit einer Gesamtfläche von $1301 \mathrm{~km}^{2}$ betroffen. Die Infektionsextensität erreichte in den Epizentren $100 \%$ und die Intensität bewegte sich größenordnungsmäßig in Tausenden von Varroa-Milben je Bienenvolk.

\section{METHODIK}

a) Die bis zum Jahresende 1982 getroffenen Maßnahmen

Sämtliche Bienenvölker wurden auf das Vorhandensein von Varroa j.-Weibchen im Wintergemüll untersucht, u.zw. mit Hilfe der Flotationsmethode in Rapsöl (spezifisches Gewicht $0,91 \mathrm{~g} / \mathrm{cm}^{3}$ ) in Mischproben aus den einzelnen Bienenstandorten (Peroutka et al, 1981). Die Gemüllentnahme aus den Windeln wurde kommissionell vorgenommen, die Untersuchungen wurden von staatlichen Veterinärinstituten durchgeführt.

Die Bienenvölker in den Infektionsherden (= Bienenstandorte) und im Umkreis von $5 \mathrm{~km}$ um den Herd wurden abgetötet. Ein Teil von der Gesamtzahl der abgetöteten Völker wurde mit Hilfe der Auswaschmethode durch 20 minütiges Ausschütteln in $55^{\circ} \mathrm{C}$ warmem Wasser untersucht. In dem durch Abtöten der Völker freigewordenen Gebiet wurden mittels der Methode « vergifteter Köder » (KAMLER et al., 1983) alle übriggebliebenen Bienen entfernt. Die giftigen Köder wurden auf einer Fläche von $1995 \mathrm{~km}^{2}$ ausgelegt. Rund um das bienenfreie Gebiet wurde eine Schutzzone von $7 \mathrm{~km}$ Breite, mit Verbot jeglicher Bienentransporte, gezogen. Nach dem Ablauf der einjährigen Beobachtungszeit wurden die sanierten Gebiete mit Bienen neu besetzt.

b) Die im Jahre 1983 getroffenen Maßnahmen

Sämtliche Bienenvölker wurden ebenso wie 1982 auf das Vorhandensein von Varroa-Milben im Wintergemüll untersucht. Völker im Umkreis von $12 \mathrm{~km}$ rund um einen Herd wurden unmittelbar nach dem Fund durch eine Besprühung mittels einer 0,01\%igen Wasseremulsion des Akarizids Taktic (Hersteller FBC, England ; Wirkstoff Amitraz $-12,5 \%$ ) in einer Menge von $40 \mathrm{ml}$ je Wabe mit der Abmessung $39 \times 24 \mathrm{~cm}$, diagnostisch behandelt. 
Die Besprühung erfolgte bei Außentemperaturen über $+2{ }^{\circ} \mathrm{C}$, besprüht wurden mit Bienen besetzte Waben nach dem Herausnehmen aus der Beute (TITĚra, 1983). Die Diagnostik wurde 12 Stunden nach der Behandlung durch Entnahme von Gemüll auf den Windeln und durch Untersuchung der einzelnen Gemüllproben als Standortmischproben durchgeführt.

Sämtliche Bienenvölker in den Varroatose-Herden, die entweder durch Untersuchung des Wintergemülls oder durch chemische Diagnose entdeckt wurden, gelangten zur Abtötung.

c) Bewertung im Frühjahr 1984

Sämtliche Bienenvölker im Umkreis von $12 \mathrm{~km}$ um die im Jahre 1983 positiven und liquidierten Herde wurden in der Winterperiode 1983/1984 mittels Besprühung durch 0,01 \%ige Wasseremulsion von Taktic diagnostisch behandelt (siehe sub b)).

Ferner wurde das Wintergemüll (Standortmischproben) aus sämtlichen Bienenvölkern in der CSR ebenso wie in den Jahren 1982 und 1983 untersucht.

Bienenvölker der positiven Standorte (Herde) wurden durch Besprühung mit 0,01\%iger Taktic-Wasseremulsion wiederholt untersucht, u.zw. aufgrund von Proben aus den einzelnen Völkern. Positive Völker wurden abgetötet.

\section{ERGEBNISSE UND DISKUSSION}

Im Verlauf der Jahre 1982/1984 gelang es, in der C'SR die erste Attacke der Varroatose zu erfassen und ihre Ausbreitung zu verzögern, bzw. an einzelnen Stellen völlig zu stoppen. Die Zahl der befallenen Bienenvölker, sowie die Extensität und die Intensität der Invasion wurde nachweisbar vermindert, wobei es gelungen ist, einen Teil der Herde zu beseitigen. Die Anwendung der radikalen Methode hat zu keiner Senkung des Gesamtbestandes an Bienenvölkern geführt.

Die Tab. 1 dokumentiert den Rückgang der Zahl der wegen Varroatose abgetöteten Bienenvölker in den Jahren 1982/1984; im Jahre 1984 verminderte sich die Zahl der positiven (abgetöteten) Bienenvölker auf $0,17 \%$ des Bienengesamtbestandes.

TAB. 1. - Zahl der wegen Varroatose abgetöteten Völker.

TABL. 1. - Number of Varroatosis killed honey bees colonies.

\begin{tabular}{l|c|c|c}
\hline \multicolumn{1}{c|}{$\begin{array}{c}\text { Jahr } \\
\text { Year }\end{array}$} & 1982 & 1983 & 1984 \\
\hline $\begin{array}{l}\text { Gesamtzahl der registrierten Bienenvölker } \\
\text { Total number of honey bee colonies registered }\end{array}$ & 604645 & 631415 & 686755 \\
\hline $\begin{array}{l}\text { Zahl der abgetöteten Völker } \\
\text { Number of colonies killed }\end{array}$ & 28264 & 3200 & 1140 \\
$\begin{array}{l}\text { \% der Gesamtzahl } \\
\% \text { of colonies killed }\end{array}$ & 4,67 & 0,51 & 0,17 \\
\hline \hline
\end{tabular}




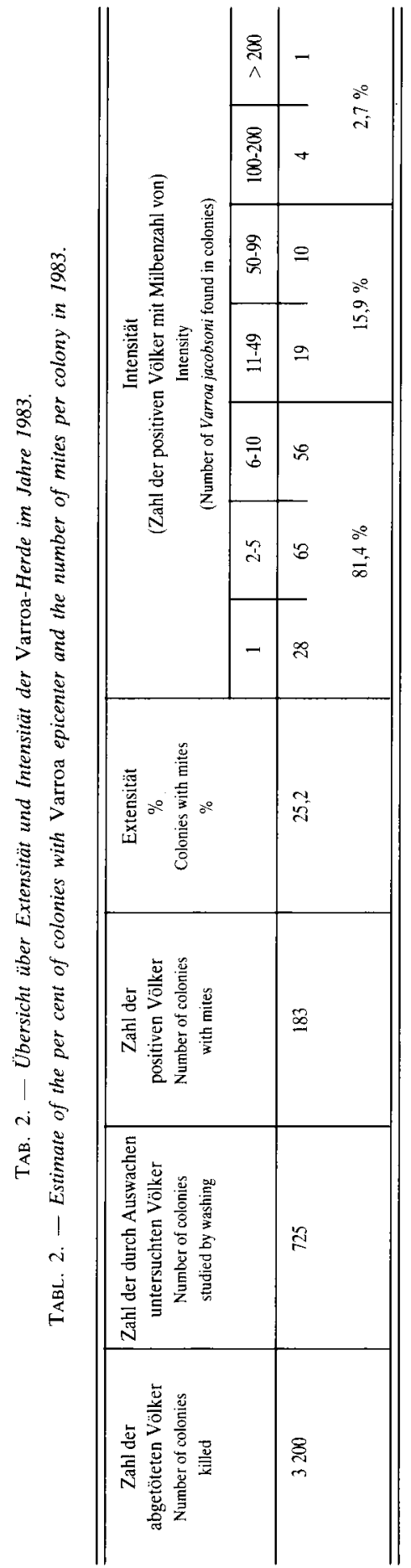


Die Tab. 2 dokumentiert den Rückgang der Extensität und Intensität der Invasion im Jahre 1983. Von der Gesamtzahl der 3200 abgetöteten Bienenvölker aus positiven Standorten (= Invasionsherde) wurden nach der Abtötung 725 Völker aus allen Gebieten des Vorkommens ( $23 \%$ aller abgetöteten Völker) mittels Auswaschen untersucht. Eine Intensität von mehr als 10 Varroa-Milben wurde nur bei $18,6 \%$ der untersuchten Völker ermittelt. In den Jahren 1983 und 1984 wurde in der ČSR kein einziger Fall des Eingehens von Bienenvölkern wegen Varroatose, sowie kein Fall von Bienenvölkern mit klinischen Symptomen der Varroatose verzeichnet.

Zur Beurteilung der Flächenausbreitung und demgegenüber der Flächeneindämmung der Varroatose führen wir eine Übersicht der Gemeinden mit dem Varroatosevorkommen in den Jahren 1981/1983 im Vergleich mit der Zahl der Gemeinden mit Varroatosevorkommen im Jahre 1984 (Tab. 3) an.

TAB. 3. - Ausbreitung und Beschränkung der Varroatose in der CSR (1981-1984).

TABL. 3. - Build-up and decline of varroatosis in the Czechoslovakia (1981-1984).

\begin{tabular}{l|l}
\hline \hline & \\
$\begin{array}{l}\text { Zahl der Dörfer mit Varroa-Herden in den Jahren 1981-1983 } \\
\text { Number of villages with Varroa epicenters from 1981-198.3 }\end{array}$ & 197 \\
$\begin{array}{l}\text { Davon : Zahl der Dörfer ohne Befund im Jahre } 1984 \\
\text { Number of villages with no report in } 1984 \\
\quad \begin{array}{l}\text { Zahl der Dörfer mit Befund im Jahre } 1984 \\
\text { villages with a report in 1984 }\end{array}\end{array}$ & 156 \\
\hline $\begin{array}{l}\text { Zahl der Dörfer mit neuen Herden im Jahre } 1984 \\
\text { Number of villages with new epicenters focus in 1984 }\end{array}$ & 101 \\
\hline
\end{tabular}

TAB. 4. - Ausbreitung der Varroa-Milben $5 \mathrm{~km}$ rund um den aus dem Wintergemüll diagnostizierten Herd.

TABL. 4. - Distribution of Varroa jacobsoni in a $5 \mathrm{~km}$ area determined by examination of bee hive winter debris.

\begin{tabular}{l|c}
\hline \hline Zahl der abgetöteten Völker & \\
Number of colonies killed & 608 \\
\hline Zahl der befallenen Völker (nach Auswaschen) & 118 \\
Number of infested colonies (after washing) & $\%$ \\
\hline
\end{tabular}

Im Jahre 1982 wurden solche Bienenvölker zur Abtötung bestimmt, die sich in einem Umkreis von $5 \mathrm{~km}$ um den Herd befanden ; die Diagnose erfolgte durch Auswaschen des Wintergemülls von 608 Vökern. Dort, wo der mittlere Befall im Herd 3,6 Milben pro Bienenvolk betrug, fiel die Gemülldiagnose der 


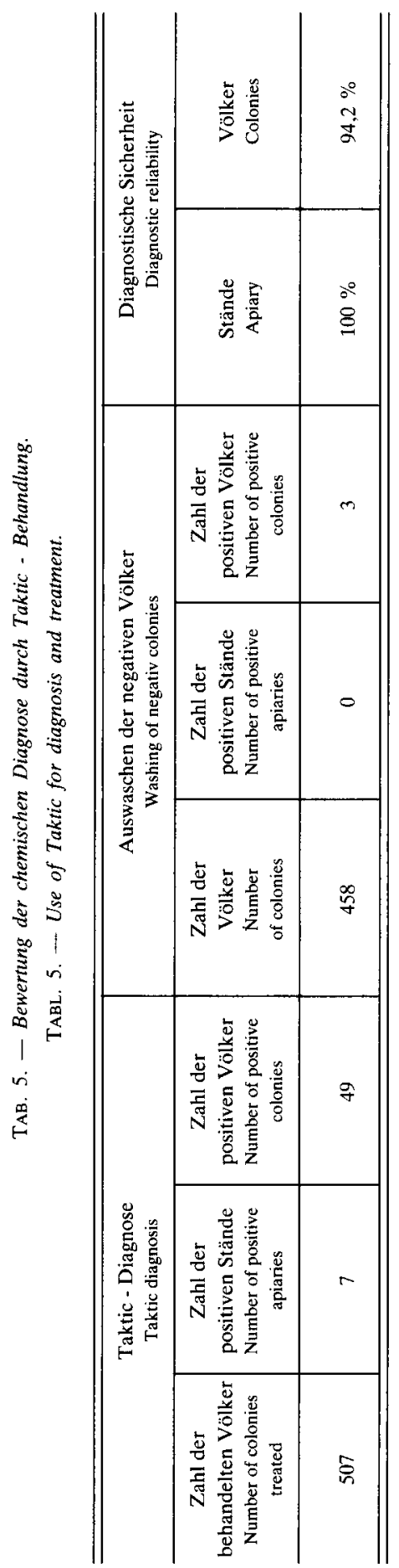


umliegenden Völker negativ aus. Insgesamt stellten wir mit dieser Methode bei den 608 Bienenvölkern einen Varroa-Befall bei $19,4 \%$ der Völker fest (Tab. 4). Dabei konnten wir im Umkreis von $2 \mathrm{~km}$ um den Herd bei $65,3 \%$ der Völker Milben feststellen, mit einem mittleren Befall von 4,9 Milben pro Volk.

Im Jahre 1983 wurden ausschließlich Bienenvölker im Varroatoseherd (= Bienenstandort) abgetötet. Diese Maßnahme ging auf Ergebnisse der im Jahre 1982 vorgenommenen Versuche mit der chemischen Diagnostik der Varroatose mittels Besprühung durch das Akarizid Taktic zurück. In den Versuchen wurden mittels Taktic $100 \%$ der Herde (= Standorte) und 94,2\% der einzelnen positiven Bienenvölker erfaßt (Tab. 5).

Das Abtöten lediglich solcher Bienenvölker, die nach der chemischen Diagnostik durch das Akarizid Taktic für positiv befunden wurden, erfolgte im Frühjahr 1984 aufgrund der Ergebnisse von Versuchen mit der Therapie der Varroatose mittels Taktic. Nach einer einzigen Behandlung der mit niedriger Invasionsintensität befallenen Bienenvölker ( $0,01 \%$ ige Taktic-Emulsion) stellten wir ein Fortbestehen der Infektion nur bei 18,1 \% der Völker fest (Tab. 6).

TAB. 6. - Therapeutischer Effekt der Taktic-Behandlung.

TABL. 6. - Therapeutic effect of Taktic treatment.

\begin{tabular}{l|c}
\hline \hline $\begin{array}{l}\text { Zahl der positiven Völker (Taktic-Behandlung) } \\
\text { Number of positive hives (Taktic treatment) }\end{array}$ & 116 \\
\hline $\begin{array}{l}\text { Zahl der Völker mit Restmilben (nach Auswaschen) } \\
\text { Number of positive residue mites (after washing) }\end{array}$ & 21 \\
& $\%$ \\
\hline
\end{tabular}

Wenn wir die Ergebnisse der radikalen Methode zur Eindämmung der Varroatose im Verlauf der zwei Jahre ihrer Anwendung in der ČSR bewerten, können wir konstatieren, daß diese Methode zumindest fähig ist, den Vorgang der Infektionsausbreitung zu verzögern und die Infektion im vorklinischen Zustand zu halten. Die Verlangsamung des Vorgangs der Varroatoseausbreitung in der ČSR ist aus den Ergebnissen von Untersuchungen des Wintergemülls im Frühjahr 1984 gut ersichtlich (Tab. 3), wo die Infektion in 156 mit Bienen neu besetzten Gemeinden, die einen positiven Krankheitsfund aus den Jahren 1981/1983 aufzuweisen hatten, nicht mehr nachgewiesen wurde und nur in 41 Gemeinden überdauerte. Das neue Auftreten der Varroatose in 101 Gemeinden ist einerseits auf eine weitere Ausbreitung aus den flächig verseuchten Gebieten aus den Jahren 1982 und 1983 und andererseits auf unerlaubtes Umtransportieren unterschlagener verseuchter Bienenvölker und ferner auf das 
Erscheinen neuer Herde in den mit den verseuchten ausländischen Regionen benachbarten Grenzgebieten zurückzuführen.

Von den 12 im Jahre 1982 flächig verseuchten Gebieten wurde die Varroatose in 6 Gebieten gedämpft. Das größte, flächig verseuchte Gebiet, in dem, sowie in dessen Umgebung, die Varroatose bereits seit 2 Jahren nicht mehr nachzuweisen war, beträgt $230 \mathrm{~km}^{2}$. Aus den übrigen 6 Gebieten breitete sich die Varroatose auf weitere benachbarte Bienenvölker aus. Es handelte sich vor allem um solche Gebiete, wo die Maßnahmen nicht konsequent genug durchgeführt wurden. Infolge gewisser Fahrlässigkeit mancher Imker und häufig auch aus Furcht vor der Behandlung der Bienenvölker unter niedrigen Temperaturen, wurden die Maßnahmen erst verspätet im Frühjahr durchgeführt. $\mathrm{Zu}$ dieser Zeit schmarotzten die Varroa j.-Weibchen an der verdeckelten Brut, entkamen so der Einwirkung des Akarizids und die schwächer befallenen Völker konnten daher nicht diagnostiziert werden. Die Durchführung der chemischen Diagnostik im Spätherbst und am Anfang des Winters ist schon deswegen notwendig, da wir in der ČSR bereits im Februar Varroa j.-Weibchen an verdeckelter Brut gefunden haben.

Die radikale Methode der Varroatosebekämpfung ist zeitlich, organisatorisch sowie finanziell recht anspruchsvoll. Als einzige vermag sie jedoch die Varroatose unter minimalem Einsatz von chemischen Substanzen zu dämmen. Chemotherapeutika werden ausschließlich zur Diagnostik angewandt und selbst dies nur in unmittelbar bedrohten Gebieten. Sie werden nur im Herbst und im Winter eingesetzt, wo die Bienen die wenigsten Waben besetzen, die Vorräte verdeckelt sind und ein verhältnismäßig langer Zeitraum zwischen der Behandlung der Bienenvölker und der Gewinnung ihrer Produkte liegt. Das Verdeckeln schützt nicht nur die Vorräte vor dem Eindringen der chemischen Stoffe, sondern diese werden mit dem Gemüll aus der Beute entfernt, selbst wenn sich diese Stoffe an das Wachs binden sollten.

Die Anwendung einer niedrigen Dosierung (6,25 mg Amitraz je Bienenvolk) zusammen mit Applikationsweise, -zeit und -organisation gewährleisten einen hygienisch einwandfreien Zustand des von den behandelten Völkern gewonnenen Honigs. Das Präparat Taktic ist in der ČSR registriert und seine Anwendung in der Bienenzucht wurde genehmigt, da im Honig der behandelten Bienen keine Residuen, weder von Amitraz noch von seinen Zersetzungsprodukten, nachgewiesen werden konnten.

Eine beträchtliche Bedeutung bei der Realisierung der radikalen Methode der Varroatoseeindämmung hat auch die Liquidation der nach der Behandlung mittels des Akarizids positiv gebliebenen Völker. Durch die Liquidation von Bienenvölkern mit überdauernder Infektion verschiebt sich wesentlich die Entstehung einer resistenten Varroa-Population. 
Erfahrungen der Versuchsperiode von 1982 bis 1984 erbrachten weitere Möglichkeiten einer Effektivitätssteigerung der radikalen Methode der Varroatoseeindämmung, die auf einer Verlagerung der chemischen Diagnostik in den bedrohten Gebieten in die Herbstperiode (Oktober) und auf der Ausnützung der Winterperiode (Dezember) zur Bekämpfung der Varroatose in den ermittelten Herden durch wiederholte Behandlung der Völker mit chemischen Präparaten (bei gleichzeitiger Beseitigung der verdeckelten Brut) und der Liquidierung von Bienenvölkern mit überdauernder Infektion beruht.

Eingegangen im September 1984.

Angenommen im Oktober 1984.

\author{
RÉSUMÉ \\ ÉVALUATION D'UNE MÉTHODE RADICALE DE LUTTE CONTRE LA VARROOSE
}

L'acarien Varroa jacobsoni a probablement été introduit en République Socialiste Tchèque au printemps 1978. Mais la maladie ne fut diagnostiquée qu'en 1981. Pendant les 3 années, durant lesquelles il n'y a pas eu de lutte, l'infection s'est étendu sur une surface de $2801 \mathrm{~km}^{2}$, i.e. $3,55 \%$ du territoire. Aux épicentres l'invasion a atteint $100 \%$ avec plusieurs milliers de femelles de Varroa.

Depuis 1981 une méthode radicale de lutte a été utilisée sur tout le territoire de la République Socialiste Tchèque. La méthode consiste à détruire le parasite avec les abeilles adultes et le couvain, à mettre en ouvre le diagnostic précoce de façon permanente et générale, à améliorer le recensement des colonies et à rechercher les colonies d'abeilles sauvages.

A la fin des hivers 1982,1983 et 1984 , on a recherché dans toutes les colonies de la République Socialiste Tchèque la présence de Varroa femelles dans des échantillons de débris hivernaux provenant de chaque rucher, par la méthode de flottaison dans l'huile de colza. En 1984 toutes les colonies, situées dans un rayon de $12 \mathrm{~km}$ autour des foyers, ont également été soumises au diagnostic chimique; les colonies ont été traitées par des pulvérisations d'une solution aqueuse à $0,01 \%$ d'acaricide Taktic (produit par F.B.C., Angleterre ; matière active : amitraz 12,5\%). En 1982 toutes les colonies des foyers ont été tuées, plus celles situées dans un rayon de $5 \mathrm{~km}$. Au total 28264 colonies (soit 4,67\% du cheptel) ont été détruites. Afin de rendre les régions totalement dépourvues d'abeilles, les abeilles des colonies non recensées ou cachées ont été attirées avec des appâts empoisonnés. Au bout d'une année d'observation, les abeilles ont été réintroduites dans ces régions. En 1983 toutes les colonies des foyers ont été tuées, ce qui représentait 3200 colonies $(0,5 \%)$. En 1984 toutes les colonies infestées ont été tuées, soit 1140 colonies $(0,17 \%)$.

La varroose en République Socialiste Tchèque a été réduite; en 1984, six ans après son introduction, la maladie a été diagnostiquée dans $0,17 \%$ des colonies avec une faible intensité — généralement jusqu'à 10 femelles de Varroa. En 1984, 156 villages et villes ont été trouvés indemnes de varroose, bien qu'il y ait eu des foyers de maladies en ces endroits entre 1981 et 1983 . On a obtenu de bons résultats avec le diagnostic chimique. Sa fiabilité est de $100 \%$ dans les ruchers et de $94 \%$ dans les colonies prises individuellement. La méthode, la période de traitement et le dosage permettent de garantir la qualité hygiénique du miel provenant des colonies traitées. La méthode permet de réduire la thérapeutique chimique et de retarder la résistance accrue des acariens.

Telle quelle, la méthode peut encore être améliorée en déplaçant la période de diagnostic à l'automne ; l'hiver peut alors être utilisé pour lutter contre la maladie en traitant plusieurs fois les colonies chimiquement, tout en ôtant le couvain operculé et en détruisant les colonies montrant une infection persistante. 


\section{SUMMARY}

\section{AN EVALUATION OF A RADICAL METHOD FOR CONTROL OF VARROA DISEASE}

Varroa jacobsoni was probably introduced into the Czech Socialist Republic in the spring of 1978. However, the disease was first diagnosed in 1981. Over the three-year period without control the infection spread to an area of 2801 square $\mathrm{km}$, i.e. $3.55 \%$ of the territory ; in the epicenters the infestation rate was $100 \%$ and its intensity reached an order of thousands of $V$. jacobsoni females.

Since 1981 the radical method of control has been used throughout the territory of the Czech Socialist Republic. The method consists of destroying the parasite together with adult bees and their brood, the enhancement of early, permanent and comprehensive diagnosis, improvement of colony recording and search for wild bee colonies.

In the late winters of 1982, 1983 and 1984, all colonies in the Czech Socialist Republic were examined for the presence of $V$. jacobsoni females in winter debris in mixed samples from each apiary by the method of flotation in rape oil. In 1983 and 1984 all bee colonies within $12 \mathrm{~km}$ of the foci were also subjected to the chemical diagnosis; the colonies were chemically treated by spraying with $0.01 \%$ water emulsion of Taktic acaricide (produced by F.B.C., England ; $12.5 \%$ amitraz as active ingredient).

In 1982 all colonies in the foci (apiary) and within $5 \mathrm{~km}$ were killed. On the whole, 28264 colonies (4.67\% of the total number) were destroyed. To make the areas entirely free from bees, bees from non-recorded and concealed colonies were attracted to poisoned baits. After one year of observation, bees were re-introduced in the areas. In 1983 all the colonies in the foci were killed. The total number of destroyed colonies was 3200 $(0.5 \%)$. In 1984 all the positive colonies were killed. The total number of destroyed colonies was $1140(0.17 \%)$.

Varroa disease was reduced in the Czech Socialist Republic. In 1984, six years after its introduction, the disease has been diagnosed in $0.17 \%$ of the colonies, with a low intensity - mostly up to $10 \mathrm{~V}$. jacobsoni females. In 1984, 156 villages and towns have been found free from Varroa disease, although there were foci in these places in 1981/1983.

Good results were obtained from chemical diagnosis of the vicinity of foci with sprays of $0.01 \%$ water emulsion of Taktic. This diagnosis has a $100 \%$ reliability in apiaries and $94 \%$ reliability in individual colonies. The method, application time and dosage guarantee hygienic safety of the honey from the treated colonies.

The method enables us to minimize the use of chemotherapeutics and delays the rise of resistance in the mites. The method can be further improved by changing the time of diagnosis to autumn. Winter is also satisfactory for controlling the disease through repeated colony treatment with chemical products while removing sealed brood and destroying colonies with persistent infection.

\section{LITERATUR}

Kamler F., VeselÝ V., Titěra D., 1983. - Objective method of removing bees from a zone for varroa disease control. XXIXth International congress of apiculture, Budapest, Hungary. Programme and Abstracts of Reports, 126.

Peroutka M., Titérova J., Haklova M., 1981. - Rapid diagnosis of varroasis from hive debris. Apiacta, 16, (3), 119.

TrTěra D., 1983. - Colony reaction to spray against varroa disease at low temperatures. XXIXth International congress of apiculture, Budapest, Hungary. Programme and Abstracts of Reports, 201. 